EESTI NSV TEADUSTE AKADEEMIA TOIMETISED. 26. KQIDE

FUOSIKA * MATEMAATIKA. 1977, NR. 2

ИЗВЕСТИЯ АКАДЕМИИ НАУК ЭСТОНСКОИ ССР. ТОМ 26 ФИЗИКА * МАТЕМАТИКА, 1977, № 2

\title{
ЗАВИСЯЩИЕ ОТ ТЕМПЕРАТУРЫ СПЕКТРЫ КОМБИНАЦИОННОГО РАССЕЯНИЯ И ФАЗОВЫЕ ПЕРЕХОДЫ В КРИСТАЛЛЕ ИОДАТА КАЛИЯ
}

K. HALLER, LJUBOV REBANE. $\mathrm{KIO}_{3}$ KRISTALLI FAASIOLEMINEKUD JA KOMBINATSIOONHAJUMISE SPEKTRITE SOLTUVUS TEMPERATUURIST

K. HALLER, LYUBOV REBANE. TEMPERATURE DEPENDENT RAMAN SPECTRA AND PHASE TRANSITIONS OF $\mathrm{KIO}_{3}$ CRYSTAL

У иодата калия, в основном по данным о диэлектрической проницаемости $\left[{ }^{1,2}\right]$, известно существование пяти кристаллических модификаций: одной высокотемпературной $\left(T_{C}=485 \mathrm{~K}\right)$ параэлектрической ромбоэдрической $\alpha$-фазы с двумя структурными единицами в элементарной ячейке и четырех сегнетоэлектрических $\beta-, \gamma-, \delta$ - и $\varepsilon$-фаз. Однако данные о структурах кристаллических решеток и даже температурах $\varepsilon-\delta, \delta-\gamma$ фазовых переходов весьма противоречивы $\left[{ }^{1-6}\right]$.

Более полно изучены триклинная $\gamma$ - и моноклинная $\beta$-фазы, хотя и здесь имеются расхождения в данных о точной структуре кристалличеткой решетки, которая согласно $\left[{ }^{3}\right]$ имеет структуру $C m$ с $Z=8$, а согласно [ $\left.{ }^{4}\right]-$ структуру $P m$ с $Z=4$. Кроме того, в $\mathrm{KIO}_{3}$ наблюдали при фазовых переходах гистерезисные явления, которые зависят от условий выращивания кристаллов [ $\left.{ }^{2}\right]$.

Сложностью объекта, по-видимому, объясняется малое число работ, где $\mathrm{KIO}_{3}$ исследуется с помощью спектроскопических методов $\left[{ }^{7-9}\right]$. Данные, полученные методом комбинационного рассеяния (KP), приведены лишь в $\varepsilon$-фазе при $T=2,7 \mathrm{~K}\left[{ }^{9}\right]$.

В настоящей работе излагаются результаты измерения спектров КР $\mathrm{KIO}_{3}$ в области температур 5-550 K. Главным образом рассматриваются изменения спектра в области внутримолекулярных колебаний при фазовых переходах.

Спектры КР регистрировались на двойном спектрометре Spex 1402 при возбуждении линией $4880 \AA$ аргонового лазера $(0,5 \mathrm{BT})$. Система регистрации $\left[{ }^{10}\right]$ включала охлаждаемый ФЭУ и автоматизированный комплекс, управляющий сканированием монохроматора, накоплением сигнала, обработкой и выдачей информации. Температура кристалла поддерживалась с точностью до $0.1^{\circ}$ в области $5-300 \mathrm{~K}$ в криостате с системой УТРЕКС, а в области $300-600 \mathrm{~K}$ - в специальной печке.

Спектры КР были зарегистрированы во всех пяти фазах. Поляризационные характеристики не изучались ввиду немоноблочной структуры кристалла. Как видно из рисунка, общий характер спектров КР не претерпевает существенных изменений при фазовых переходах. Во 


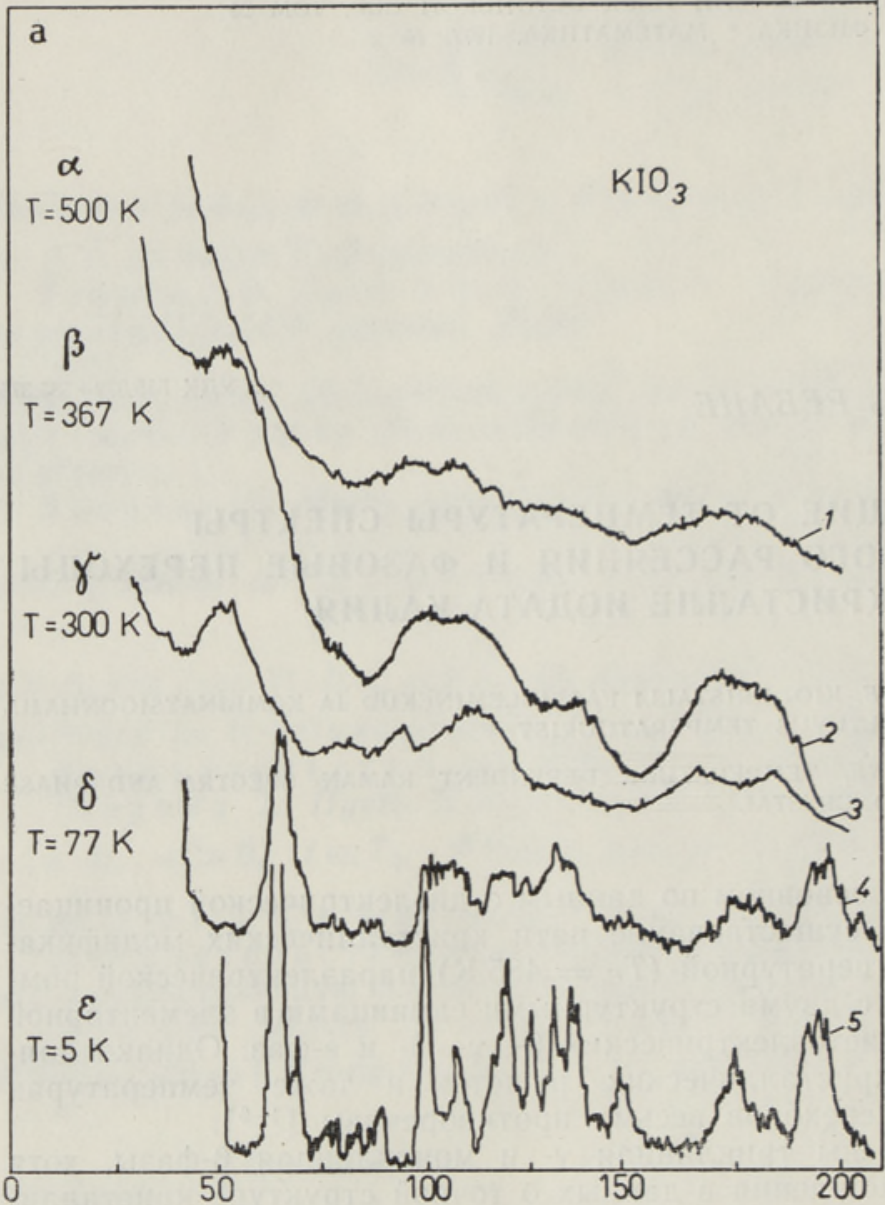

Спектры КР кристалла $\mathrm{KIO}_{3}$ в области кристаллических $(a)$, внутримолекулярных деформационных (б) и внутримолекулярных валентных (в) колебаний в $\alpha$-, $\beta-, \quad \gamma^{-}, \delta$ - и $\varepsilon$-фазах.

всех фазах спектр содержит хорошо разделенные три области: область валентных колебаний $\mathrm{IO}_{3}^{-} 700-820 \mathrm{~cm}^{-1}$, область деформационных колебаний $\mathrm{IO}_{3}^{-} 300-380 \mathrm{~cm}^{-1}$ и область внешних решеточных колебаний до $200 \mathrm{CM}^{-1}$.

Обращает на себя внимание сходство спектров в области валентных и деформационных колебаний. Здесь наблюдается по пяти групп линий (обозначены на рисунке буквами $A, B, C, D$ и $E$ ), которые происходят из двух валентных $v_{1}(A), v_{3}(E)$ и двух деформационных $v_{2}(A)$, $v_{4}(E)$ колебаний $\mathrm{IO}_{3}^{-}$.

Рассмотрим поведение этих групп линий при фазовых переходах. Наиболее интенсивной является группа $A-B$ линий в валентной области. В $\delta$-фазе она имеет наиболее сложную структуру - выделяются пять максимумов при $727,733,739,744$ и $751 \mathrm{~cm}^{-1}$. В $\varepsilon$-фазе структура несколько упрощается - исчезает пик при $744 \mathrm{~cm}^{-1}$, сужаются линии 728 и $752 \mathrm{CM}^{-1}$. При $\delta-\gamma$ фазовом переходе $A-B$ линии сдвигаются на 8-10 $\mathrm{cm}^{-1}$ в высокочастотную сторону и в высокотемпературных фазах не обнаруживают видимой структуры. В деформационной области $A$ и $B$ линии в $\varepsilon$ - и $\delta$-фазах узкие и, по-видимому, элементарные. В остальных трех фазах $A$ линия отсутствует, а $B$ линия уширяется и имеет неразрешенную структуру. 

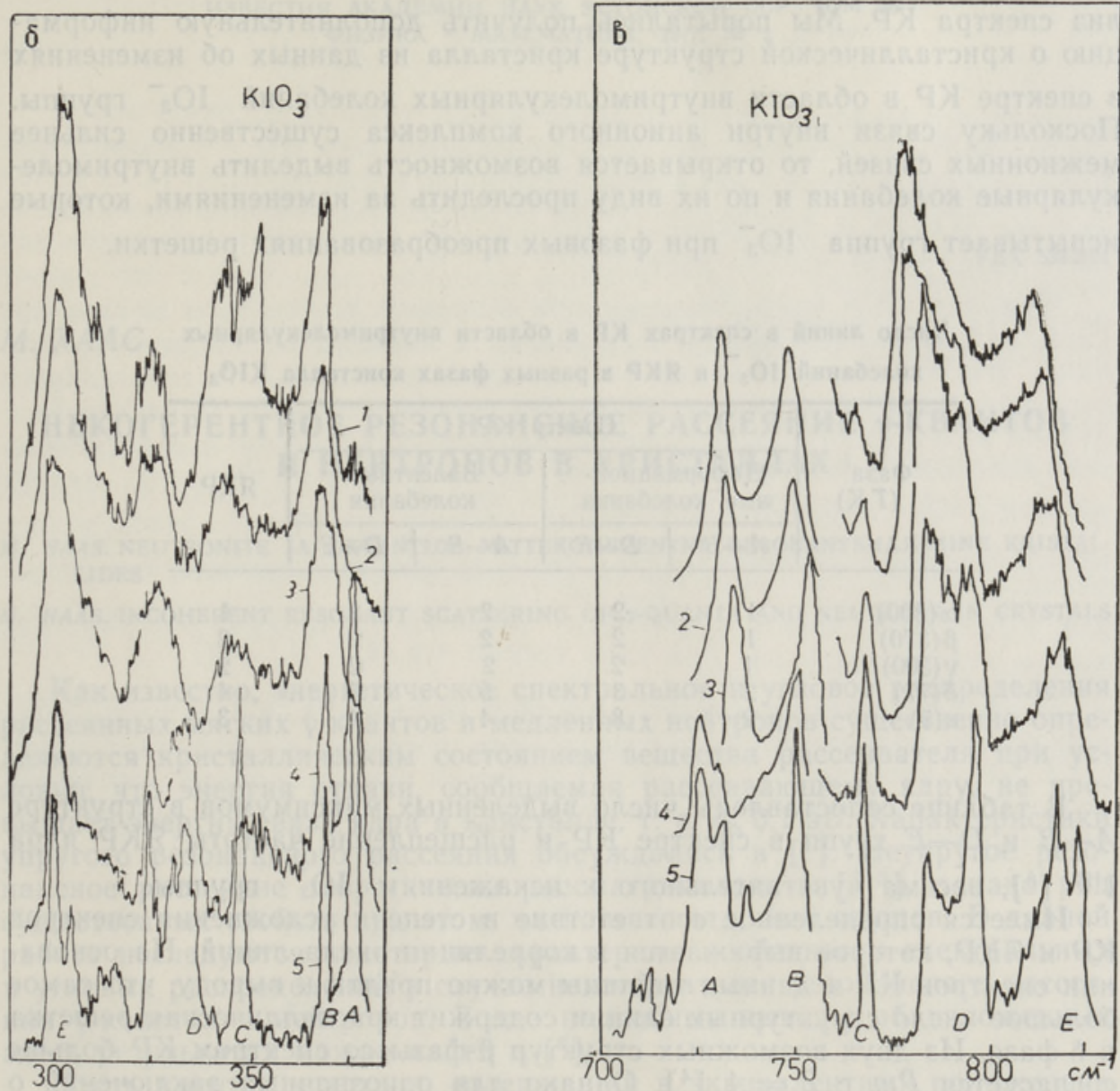

Группа $D-E$ линий обладает наиболее сложной структурой также в $\delta$-фазе - видны по крайней мере пять максимумов при 784, 787, 796 и $813,816 \mathrm{~cm}^{-1}$. В $\varepsilon$-фазе выделяются четыре линии 789,796 и 816, $823 \mathrm{~cm}^{-1}$, а остальные значительно ослабляются. В $\alpha$-, $\beta$ - и $\gamma$-фазах наблюдаются два широких максимума без заметных изменений. Аналогичные два широких $D-E$ максимума имеются в высокотемпературных фазах и в деформационной области. В $\delta$-фазе просматриваются два широких 302 и $335 \mathrm{~cm}^{-1}$ и один узкий $326 \mathrm{~cm}^{-1}$ максимумы, а в $\varepsilon$-фазе их число равно восьми.

Наиболее резкие изменения при фазовых переходах испытывает $C$ полоса. В $\varepsilon$-фазе это узкая элементарная линия. В валентной области интенсивность $C$ линии с ростом температуры постепенно падает и в $\alpha$-фазе виден только ее след. В деформационной области эта линия исчезает уже в $\delta$-фазе и на ее месте появляется широкая маломеняющаяся полоса.

Картина изменения спектров КР при фазовых переходах усложняется еще и тем, что температурное равновесие в $\mathrm{KIO}_{3}$ достигается за большие промежутки времени, что приводит к явлениям типа гистерезисных.

Недостаточность сведений о симметрии кристаллической решетки $\mathrm{KIO}_{3}$ в низкотемпературных фазах затрудняет последовательный ана- 
лиз спектра КР. Мы попытались получить дополнительную информацию о кристаллической структуре кристалла из данных об изменениях в спектре КР в области внутримолекулярных колебаний $\mathrm{IO}_{3}^{-}$группы. Поскольку связи внутри анионного комплекса существенно сильнее межионных связей, то открывается возможность выделить внутримолекулярные колебания и по их виду проследить за изменениями, которые испытывает группа $\mathrm{IO}_{3}^{-}$при фазовых преобразованиях решетки.

\begin{tabular}{|c|c|c|c|c|c|}
\hline \multirow{3}{*}{$\begin{array}{l}\text { Фаза } \\
(T \mathrm{~K})\end{array}$} & \multicolumn{4}{|c|}{ Спектр КР } & \multirow{3}{*}{ ЯKP } \\
\hline & \multicolumn{2}{|c|}{$\begin{array}{l}\text { Деформащион- } \\
\text { ные колебания }\end{array}$} & \multicolumn{2}{|c|}{$\begin{array}{l}\text { Валентные } \\
\text { колебания }\end{array}$} & \\
\hline & $A-B$ & $D-E$ & $A-B$ & $D-E$ & \\
\hline $\begin{array}{l}\alpha(500) \\
\beta(370) \\
\gamma(300) \\
\delta(77) \\
\varepsilon(5)\end{array}$ & $\begin{array}{l}1 \\
1 \\
1 \\
2 \\
2\end{array}$ & $\begin{array}{l}2 \\
2 \\
2 \\
3 \\
8\end{array}$ & $\begin{array}{l}2 \\
2 \\
2 \\
5 \\
4\end{array}$ & $\begin{array}{l}2 \\
2 \\
2 \\
5 \\
4\end{array}$ & $\begin{array}{l}1 \\
2 \\
2 \\
4 \\
3\end{array}$ \\
\hline
\end{tabular}

В таблице сопоставлены число выделенных максимумов в структуре $A-B$ и $D-E$ групп в спектре КР и расщепление частоты ЯКР ядра $\mathrm{I}^{127}\left[{ }^{8}\right]$, весьма чувствительного к искажениям $\mathrm{IO}_{3}^{-}$группы.

Имеется определенное соответствие в степени усложнения спектров КР и ЯКР, которое выражается в корреляции числа линий. На основании спектров КР и данных таблицы можно придти к выводу, что самое большое число структурных единиц содержит красталлическая решетка в $\delta$-фазе. Из двух возможных структур $\beta$-фазы со спектром КР больше согласуется $P m$ с $Z=4\left[{ }^{4}\right]$. Однако для однозначных заключений о числе неэквивалентных групп в элементарной ячейке необходимы дополнительные исследования.

\section{Л ИТЕРА Т Р А}

1. He r 1 a c h, F., Helv. Phys. Acta, 34, 305 (1961).

2. S a 1 j e, E., Z. Kristallogr., 134, 107 (1971).

3. H a m i d, S. A.. Z. Kristallogr., 137, 412 (1973).

4. S a 1 j e, E., Z. Kristallogr., 137, 1 (1973)

5. Шувалов Л. А., Иванов Н. Р.. Чихладзе О. А., Изяаиленко А. Н., Кристаллография, 18, 1207 (1973).

6. Hel g, U.. Z. Kristallogr., 131, 241 (1970).

7. Б а й с а Д. Ф. и др., УФЖ. 17, № 8, 1346 (1972).

8. Б а й с а Д. Ф. и др., Изв. АН СССР. Сер. физ., 39, № 4, 702 (1975).

9. S a 1 j e, E., Z. Kristallogr., 139, 317 (1974).

10. Ребане Л. А.. Хальдре Т. Ю., Новик А. Е., Гороховский А. А., ФTT, 15,3188 (1973). 